\title{
Bio-mechanism response of Mimosa pudica against external stimulation
}

\begin{abstract}
Mimosa Pudica (also known as Pokok Semalu) is an action plant with unique biological cell mechanism that has great potential to be explored for next generation biomechatronics devices. The motion principle of each petiole movement occurs by an organ of motion, called pulvinus. The behavior analysis of the Mimosa Pudica plant main pulvinus and petiole against external stimulations has been investigated. The response of the plant cell was observed by optical and scanning electron microscopes (SEM). External stimulation tests on Mimosa Pudica plant such as mechanical, electrical and light stimulations were conducted. Optical microscopy and SEM observations revealed the existence of red cells in the tertiary pulvinus. Mechanical stimulation results had shown a response time of approximately 2 seconds and a recovery time of 10 to 12 minutes for the leaves and 20 minutes for the petiole. Bending force of the petiole of Mimosa Pudica was also measured. The torque generated by the bending of petiole was found to increase exponentially as the pulvinus diameter increased. A torque of $30.91 \mathrm{~g} \mathrm{~mm}$ was generated from the bending of a petiole with $1.5 \mathrm{~mm}$ pulvinus diameter. The lifting potential of the Mimosa Pudica was tested using loadings with increments of 0.42 gram. The plant responded to electrical stimulation of 1.3 Ampere and displayed side effect. The light sensitivity region of Mimosa Pudica was obtained through light stimulation using a full spectrum daylight bulb, and ranges from 50 to 300 lux. Signal transmission to neighbouring structures was observed when the leaf was stimulated with threatening stimulus, with the signal speed achieving $0.8 \mathrm{~cm} / \mathrm{s}$. Movement mechanism of Mimosa Pudica is believed to be triggered by signal through receptor cells. It was found that the torque generated during petiole lifting is relatively higher than that during petiole bending.
\end{abstract}

Keyword: Microscopy; Mimosa pudica; Movement; Pulvinus; Stimulations 